\title{
Publisher's Note: Production of high-quality electron beams in numerical experiments of laser wakefield acceleration with longitudinal wave breaking [Phys. Rev. ST Accel. Beams 6, 121301 (2003)]
}

\author{
P. Tomassini, M. Galimberti, A. Giulietti, D. Giulietti, L. A. Gizzi, L. Labate, and F. Pegoraro \\ (Received 30 December 2003; published 16 January 2004) \\ DOI: 10.1103/PhysRevSTAB.7.019901 \\ PACS numbers: 52.38.Kd, 52.65.Rr, 99.10.Fg
}

This paper was published online on 30 December 2003 with an incorrect page number in Ref. [21], resulting in the link to the cited article failing. The error has been corrected as of 30 December 2003. 